\title{
Chapter 7 \\ Data-Related Ecosystems in Policy \\ Making: The PoliVisu Contexts
}

\author{
Giovanni Lanza
}

\begin{abstract}
The article explores the complexity of the ecosystems that develop around data supported policy making. This complexity, which can be traced back to the multiplicity of actors involved, the roles they assume in the different steps of the decision making process, and the nature of the relationships they establish, takes on new connotations following the rising use of data for public policies. In fact, issues related to data ownership and the ability to collect, manage, and translate data into useful information for policy makers require the involvement of several actors, generating ecosystems where co-creation strategies are confronted with the limits of action of the public administrations within broader social and decisional networks. Based on this background, the article aims to provide, through the analysis of the direct experiences conducted by the pilot cities involved in the PoliVisu project, an overview of the opportunities and challenges related to the impact of data in the evolution of decision making networks and ecosystems in the data shake era.
\end{abstract}

Keywords Actor-network theory · Data supported policy making · Digital transformation $\cdot$ Decision making ecosystems

\subsection{Introduction}

Thanks to the development and the widespread diffusion of information and communication technologies (ICT), contemporary cities produce an increasing amount of digital data. This unprecedented availability of information about the behaviours, choices, needs, and desires of large samples of individuals collected on extended time frames allows the adoption of a holistic perspective on emerging urban dynamics in a way that can deeply innovate how public policies are constructed (Rabari and Storper 2015; Kitchin 2014a, b). The literature focuses on this opportunity, underlining the possibility, introduced by the constant availability of digital data, to conceive the policy making process as a continuous experimental activity. In this framework, the

G. Lanza (®)

Department of Architecture and Urban Studies, Politecnico di Milano, Milan, Italy

e-mail: giovanni.lanza@polimi.it 
approach to a public issue is based on the diagnosis of current trends as a starting point to design future scenarios. Then, the policy response is dynamically implemented, and its impact is evaluated by considering, through the analysis of data-based feedback, the reactions and effects produced by the policy itself. Thanks to the ongoing data revolution, decision makers can thus not only rely on an increasing analytical capacity which enables them to shed light on the complex dynamics which involve contemporary cities and their populations, but also to respond more effectively to emerging issues through the implementation of targeted public policies.

However, it is essential to point out that the opportunity to promote institutionalized data-supported policy making processes entails several difficulties whose magnitude is directly proportional to the complexity of the interactive network of public and private actors involved and, clearly, to the complexity of the problems to be addressed.

Among the possible challenges facing public administrations, two seem particularly relevant to this article.

The first challenge concerns the possibility (or the willingness) of a public actor to experiment with the use of digital data to drive its decision making activities (Thakuriah et al. 2017). This opportunity is influenced both by the actor's propensity to innovate and by the position the actor occupies in the institutional framework of reference: a variation in the availability of political and economic resources, that are both necessary in the field of digital innovation, can generate potential imbalances between administrations.

A second challenge concerns the ability to structure a culture of datacy, which, in Batini's words (2018), is a measure of the decision makers' capability to collect different data, evaluate its quality, interrogate, and use it to analyse reality and solve complex problems. This perspective is based on the ability (and the necessity) to build political, technical, and legal frameworks to let individuals and government entities to be able, for each phase of the policy making process, to collect, access, produce, and analyse data-based information of which the production and ownership are increasingly fragmented. Thus, cities face the growing need to involve additional actors and expertise, from data owners to technical partners, ultimately increasing the level of institutional complexity of the data-related ecosystems.

The two challenges underline the importance and the need for a behavioural change on the part of decision makers who, due to the effects of the data shake, find themselves involved in increasingly complex and ramified decision making networks. Within these ecosystems, whose level of complexity is linked to the content and relevance of the policy in question, the opportunities and challenges introduced by the data bring the participating actors, some of them new but with increasingly central roles, to interact with each other in new and dynamic ways.

Therefore, public administrations are required to develop the ability to build and manage these ecosystems by limiting potential conflicts between stakeholders and adopting strategies to deal with some critical aspects related to digital innovation, such as the problems of data ownership and the dependency on providers of data and technologies. 
In this article, some characteristics of these ecosystems are analysed, with particular attention to the new types of participant actors and to the new data-oriented relations they establish, referring to the experiences of the pilot cities of the PoliVisu project that have been directly confronted with this relevant effect of the data shake.

\subsection{The PoliVisu Project as a Testbed for Digital Innovation}

The PoliVisu project, developed within the European Horizon 2020 program framework, offers an interesting case study to analyse and understand the opportunities and challenges of digital innovation in urban governance. PoliVisu, in particular, aims to test the usefulness of data visualisations, collected from different sources, and related to multiple urban issues, in supporting public sector's decision making activities. To achieve this aim, in the awareness of the technical-political implications associated with the nature of these experiments, the project promotes the establishment of a system of relationships and mutual support by including actors with different expertise and competences in the PoliVisu consortium.

In this macro-network, the pilot cities' administrations have played the most relevant role for the topic of the present article, mediating between the general objective of the project and the difficulties inherent in dealing with the contextual and concrete promotion of data supported policy making they have experimented. To face the challenges they encountered while implementing their activities, partners have built local micro-networks, involving both other members of the consortium and external local actors to respond, with a data and visualisation-based approach, to the main policy questions arising in their specific contexts.

Thanks to the analysis and mapping of the activities of the pilot cities it has been possible to paint a policy network canvas for each municipality, whose characteristics are discussed in the following sections of the article.

This analysis has allowed us to capture to what extent each city took the opportunity to use the resources and the context of PoliVisu to test a new and different approach to policy making and governance. The identified ecosystems, which are flexible and evolving networks of relationships, exist and change according to the emerging public issues to be faced. In fact, the contents of a policy affect the type of actors to be involved in the network and the choice and availability of data to collect, analyse, and visualise. This condition has emerged in the PoliVisu project, where the pilot cities differ in size and in their position in their administrative framework of reference. Moreover, they have been confronted with emerging local issues that, although primarily related to mobility, have been tackled differently due to the different availability of data and the influence of the local and supra-local political agendas, thus leading to the construction of networks of varying complexity.

Five pilots are participating in the PoliVisu project. The case of Issy-lesMoulineaux (France) concerns a medium-small city of the Paris metropolitan region affected by high levels of car congestion. This condition is mainly due to the city's 
economic vitality and commuting transit to and from Paris. Therefore, Issy-lesMoulineaux wishes to communicate real-time traffic information to citizens and develop a control dashboard to support the public administration's operative sectors in their planning activities. The Issy pilot's primary goals are related to establishing an effective and clear communication with the citizens (to achieve a long-term effect of behavioural change in mobility habits) and to test how data visualisations can support decision making processes and facilitate collaboration within the administration.

A different situation characterizes the pilot of Ghent (Belgium), which aims to identify the hidden population of students. Every year many unregistered students reside in Ghent, widely influencing the housing market and the mobility system. Identifying where students live can help the policy making process and produce positive and relevant impacts on the city's liveability.

Also, the pilot of Pilsen (Czech Republic) is mainly focused on mobility. The layout of the city causes several congestion challenges and problems. To monitor and predict the impact of changes, such as roadworks, the traffic model developed for the city will be improved during the project using traffic sensor data to identify and visualise traffic volumes and their changes over time.

The Mechelen pilot (Belgium) has two main objectives. The first objective is to provide traffic modelling for the city. Mechelen, together with the police zone Mechelen-Willebroek, has integrated an existing traffic model and aims to enrich its usefulness by adding data from ANPR (Automatic number-plate Recognition) cameras. The traffic model will be used as a predictive tool to monitor the impact of planned road works in the city. The second objective deals with the recently introduced "school streets" where traffic calming solutions such as road closures are in place. The aim is to measure and analyze traffic and congestion variation (both in the closed streets and the neighboring streets) before and after implementing the measure.

Finally, the Flanders pilot (Belgium) concerns the traffic accident map, a tool that visualises the traffic accident data which is obtained from the federal police, allowing specialized analytics directly on map (through heatmaps and application of advanced filters).

It can be noted that the pilot cities are very different both in terms of the issues they deal with and in the roles played by the involved actors within the decisional ecosystems. However, the analysis of the activities and networks built by the pilots, which is presented in the following sections of this article, shows that evident similarities can be identified in the challenges they face and the strategies they are putting in place to tackle them.

The mapping of the activities and networks that have developed thus becomes a valuable resource both during the implementation of the experiments and in a subsequent phase of process evaluation to manage and understand the complex decision making ecosystems of the digital age. 


\subsection{Actors and Roles in Data-Related Policy Making Ecosystems}

In the field of public policy analysis, the social network that develops around any policy decision represents a fundamental object of study. In fact, the reconstruction of the stages of a decision making process allows us to understand how the participant actors, the relationships they establish, and the resources they spend, have contributed to achieving a certain outcome, namely, the public decision that is the object of analysis (Dente 2011). Although this analytical technique seems more suitable to study processes in a posteriori perspective, therefore considering decisions that have already been taken, it is still interesting to try to apply it in the context of PoliVisu and the pilots' activities, where the experimental nature of the project introduces an additional element of complexity. In fact, the challenges faced by pilots in their path have led to a deeper reflection on their internal organization and the possible evolution of their activities and role in the era of digital innovation.

As a result, it seems reductive to analyse the cases starting from the assumption that the pilots have linearly shaped their activities by focusing only on the initial problem throughout the process. Rather, starting from a specific issue that would have represented an interesting field of data and visualisation experiment, the pilots have generally organized their own micro-network and changed their behaviour to respond to the difficulties they encountered in progressing their activities.

For this reason, the analysis of the pilot cities' networks is related to processes that are still ongoing where the local public administrations keep on experimenting with new kinds of interactions and opportunities. In this perspective, the experimentation becomes a useful approach to guide the public sector in the transition to the digital age acquiring, in the process, a new awareness of its role and responsibilities.

The analysis carried out for the PoliVisu cases is mainly based on the model proposed by Dente (2011) but introducing some changes to adapt the model to the innovations brought by the data shake on decision making ecosystems. Among these, one of the most important appears to be the widening of the range of actors involved. Establishing an agile and effective data supported policy making is an interdisciplinary challenge that requires the co-presence of multiple perspectives. These different viewpoints are related to specific competencies that are not easily found in one multi-talented person. PoliVisu's experience confirms that, in the digital age, the presence and collaboration of different expertise and the availability of multiple viewpoints becomes an essential resource (Wiliford and Henry 2012) to create an environment in which decisions and the use of data to support them can be developed in an integrated and effective way. Through participant observation and interviews, the actors that each pilot has involved have been identified according to their roles within an actor-network scheme and organized into four categories, which should not be considered impermeable; each actor, in fact, can play different roles in the process, thus contributing to making these ecosystems even more complex (Fig. 7.1). 


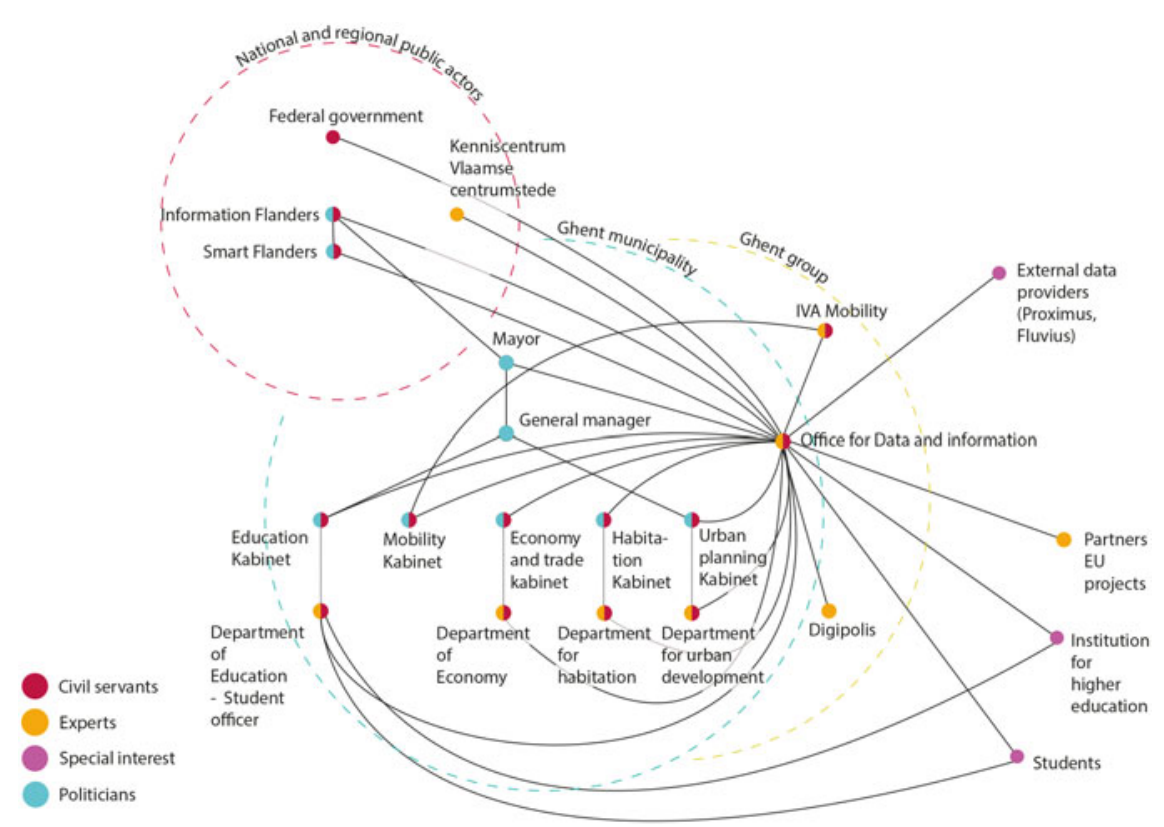

Fig. 7.1 Example of the actor-network from the case of Ghent

Politicians justify their actions based on available political resources given by their status of elected representatives of citizens. In the PoliVisu pilot's case, each context shows different configurations of the participation of politicians in the decision making network. This outcome is due to the fact that the cities involved represent different countries, each one characterized by its specific structure and culture of governance and policy objectives that may or may not facilitate the promotion of digital innovation paths at the municipal level. Although the pilot cases are very different from each other, the analysis shows that regardless of the level of autonomy of each city in promoting its experimental approach to data supported policy making, the role of supralocal policy actors is relevant since it can enable and facilitate, through the definition of regulatory frameworks, the experimental data-oriented activities of local governments.

Many of the political actors identified in the networks represent complex and diverse organizations. It happens that, for example, the same organization can take on both a political and administrative role. Therefore, all those actors who actively participate in the functioning of the administrative machine and whose action is bound by political guidelines and legal frameworks have been indicated as civil servants. In PoliVisu and other data-related ecosystems, a central role is assumed by the offices for data and information (smart city department). Their task is to facilitate digital innovation by continually mediating between the inputs of political actors, the needs of other public administration members, and the proposals and requests of external partners, such as data providers and technical experts. The civil servants of a smart 
city office are essentially dynamic figures working in a multidisciplinary environment, in line with the evolution of governance in the era of digital innovation. Their role requires a specific ability to interact with other public administration sectors, responding to their requests and facilitating their activity through a continuous ability to communicate with external partners that provide data and services that the public administration does not own.

These interactions are supported by the third type of actor identified during the analysis, the experts, who possess specific skills or knowledge resources that can be spent in policy making processes. Since public administrations are transforming into data-driven organizations, many of the expertise needed to extract value from data may be available within the structure, and it is for this reason that in the decisional ecosystems of the PoliVisu pilots central actors such as the smart city offices take on at the same time the role of civil servants and experts. However, as shown by the PoliVisu cases, referring to external actors is necessary when this expertise is not available within the organization or when it comes to access to data not directly produced or owned by the public sector.

Indeed, if in the past we could argue that the public sector was holding a large number of datasets to be opened, today we have a growing number of high-value datasets held by private bodies and not being used for a public will. To obtain this data, public authorities need to negotiate and buy it from private companies. External data providers are thus becoming an increasingly important player within decision making ecosystems.

They fall within the fourth and final category, the one of the actors with special interests. The actors that play this role participate directly or indirectly in the process for two main reasons.

The first is linked to the fact that the policy decision in question may affect their interests. This mechanism may lead either to the actor's direct participation in the decisional arena or by influencing (both directly and indirectly) the other actors involved in the construction of the policy. The latter option happens, for instance, with citizens or other policy recipients/targets that may express their favour or opposition to a policy through public opinion and voice.

The second is related to the fact that actors representing special interests can build, by participating into the policy making arena, further opportunities beneficial for their interests, as happens in the case of data and service providers interested in collaborating with public administrations to promote their business, build know-how and gain visibility. The relationship with these actors, which are generally external to the organization but fundamental to access data, requires both the existence and adoption of procurement frameworks and good negotiation skills from the public sector.

The actors and roles that have been identified are the results of a generalization based on the PoliVisu pilots' experience. Observing how cities have extended through their experimental activities these ecosystems even beyond the borders and the actors of the project, it is thus demonstrated that digital innovation challenges are faced by involving several and different actors and expertise. However, it should be stressed that an expansion of the network can, in turn, introduce new challenges 
that cities must face. In fact, too complex networks featuring a wide variety of points of view expressed by different actors, or very dense ones characterized by a large number of relationships involving at the same time several actors, tend to be less manageable and limit the chances of a successful decision making process. Pursuing a balance between the expansion of the network and its sustainability is one of the great challenges introduced by the data shake that is probably engaging the pilots of PoliVisu and many other cities in the world.

\subsection{Data-Related Relations}

Digital innovation in policy making requires the presence and joint action of multiple actors and expertise, leading to the consequent expansion of decision making ecosystems. However, the increasing complexity of these processes is due to the growing number of players involved and the nature of the interactions they establish. In this context, the involvement of new actors is functional in developing new and profitable relationships necessary to overcome problems and challenges induced by data and visualisation use in policy making processes. By operating an abstraction, decision making ecosystems can be seen as networks of nodes and lines. Each node represents an actor who, as we have seen previously, takes on one or more roles depending on the interest or duty that drives its participation in the process. Moreover, according to the role it assumes, each actor possesses certain resources that can be economic, political, cognitive, legal, and relationship based. Therefore, the participation and behaviour of each actor within the network will be influenced by the availability of resources and whether the same actor is willing to spend them to achieve its objectives. Consequently, the interaction between actors can essentially be interpreted as an exchange of resources between participants in the same decision making process. The lines connecting the different nodes of the network become, ideally, conduits through which these resources can flow, setting in motion the whole interactive mechanism that gives life to the ecosystem.

In the era of the data shake, networks extend and become more complex because the challenges related to data collection, management, and analysis require the availability of more resources and the involvement of multiple actors who can share them. For this reason, analysing the interactions between the participants of a decision making process and their evolution in the digital age allows us to reconstruct the participation strategies of the actors and understand how they face the challenges encountered in the innovation path.

From this point of view, the PoliVisu pilots represent interesting and concrete cases to analyse these interactive mechanisms that were mapped in the actor-network grouping the types of interactions that occur in six categories (Fig. 7.2).

However, it is worth noting that this categorization introduces a simplification since data-related activities in the network can lead the same actor to interact with each other for different reasons and, consequently, exchange different resources according to the multiple roles it can play. Furthermore, the six categories describe 


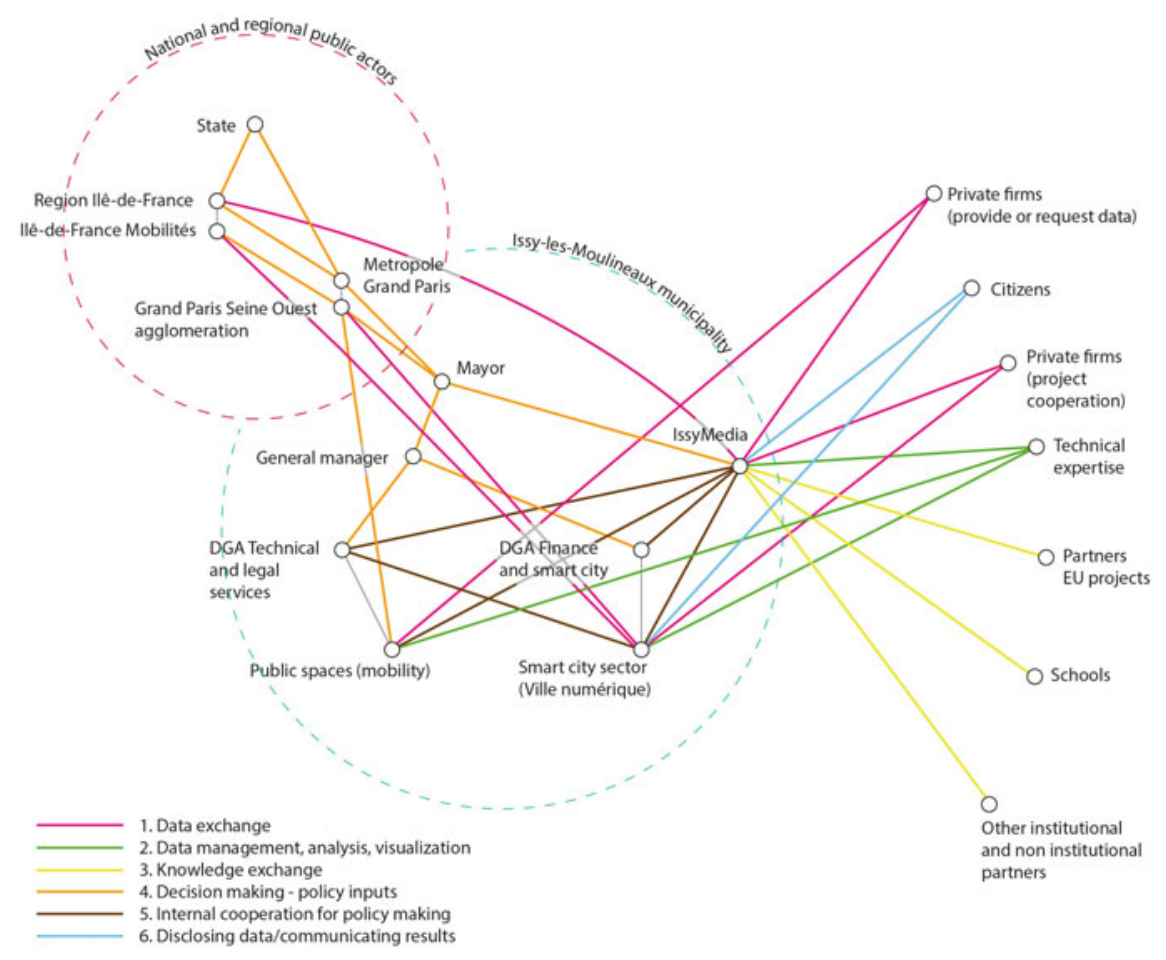

Fig. 7.2 Example of the relations occurring in the actor-network from the case of Issy-lesMoulineaux

interactive processes taking place within most of the pilot cases, underlining how similar kinds of relationships can be established in different contexts.

The first category of relationships, data exchange, refers to the transfer of data between different actors that, in the cases of PoliVisu, generally takes place internally or externally to the public administration that is centrally involved in the experimentation.

In the case of internal exchanges, it is possible that an actor requests data that are produced and owned by other sectors of the same organization. The quality of these interactions strongly depends on the level of datacy of the organization itself, as it affects the efficiency of the internal functioning and the regulatory frameworks that distribute the competencies in data production and sharing. For example, it can be observed that these exchanges can be problematic in the typically siloed structures of municipalities where the different sectors are often not well connected between them. In this sense, the data produced or stored by one of the silos is considered a sort of exclusive property and is not shared, even if that may benefit the municipality. Siloed organizational status is reproduced in data production and management.

Such limits can be encountered both within the same organization and in the relations between public organizations belonging to different administrative levels 
and with different competences. In these cases, therefore, we speak of exchanges that happen externally to the reference organization, which also increasingly involve actors that are not part of the public sector. As seen in the previous section of the article, this type of relationship, which requires considerable negotiation skills and raises important ethical-legal issues, is often essential to access the data needed to support policy making activities.

The second category concerns the relationships established between actors with different expertise to manage, analyse, and visualise data. Certain tasks are distributed through these relationships that make the actors responsible for more technical or advanced data storage, analysis and visualisation processes. By involving different points of view, which can be represented by subjects both internal and external to the organization, it is possible to obtain extremely relevant support to carry out the policy-oriented data activities that allow exploiting the value of data in decision making processes.

The third category concerns the exchange of knowledge. In the cases of PoliVisu these relationships are in place when the network features actors participating in the process to share know-how and adopt a best practice approach as happens, in particular, with the partners of the project consortium. Simultaneously, a different form of knowledge can be transmitted by local actors, not only in the form of data about issues within their competence but also through a greater ability to intercept citizens' needs and bring them back into the decision making arena. These actors play an important consultancy role that can significantly increase the effectiveness of data supported decision making mechanisms and the quality of the content of a policy.

The fourth category introduces another central issue of policy making and concerns the impact of political guidelines on decision making processes. The label policy inputs - decision making identifies the power relations that typically emerge between political actors and civil servants. In the case of policy inputs, the former, using resources linked to the role attributed to them by popular consensus, have the power to establish the political agenda, pointing specific issues of public interest and orienting the consequent policy responses. They also have the power to establish legal frameworks to regulate the use of data for innovation policies, thus guiding the activities of the operative sector of the public administration.

On the other hand, this relationship may also concern the transmission of data and visualisations in the opposite way, from the operational sectors of public administration to the policy makers. This opportunity is extremely relevant since it can become a key strategy to promote and enhance the efficiency of decision making activities.

Important, in this sense, is also the possibility to use data and visualisations to facilitate interactive processes between members of different operational sectors within the same organization. The fifth category identifies these relationships as internal cooperation for policy making, which includes all relationships and exchanges of resources intended to facilitate the sharing of data, information, and knowledge between different sectors, increasing their permeability and capacity for interaction. Although each operational sector of public administrations is generally responsible for some public issues according to a siloed logic, promoting modes of organic 
action and cooperation can be a way to make the organization's activity more efficient, thus avoiding the waste of valuable resources and promoting, at the same time, a datacy-oriented approach.

Finally, a last category of relations can be established between the public administration and the recipients of the policy decision, through disclosing data and communicating results. Especially in the case of Issy-les-Moulineaux, the digital revolution taking place in the municipality is interpreted as a valuable opportunity to strengthen the link with citizens providing, under the form of open data, both relevant information that is useful to organize their everyday life and insights about the activities of the municipal administration. Moreover, this type of relationship can be configured, depending on the intentions and the regulatory framework of each context, as the result of a strategy of direct involvement of citizens in the decision making arena, legitimizing their participation as representatives of special interests affected by the policy decision. However, engaging the public in policy making is one of the most significant challenges public administrations struggle with. For this reason, although in PoliVisu pilots this type of relationship is present only in a few cases, it can assume great relevance in the digital age. In fact, thanks to the development and diffusion of ICTs, there is an increasing interest in the citizens' broader involvement both as data providers and as contributors in decision making processes (Calzada 2018). Thus, public administrations should identify strategies to better structure and develop these relations which may be crucial for further developing digital innovation.

\subsection{Conclusion: Dealing with Complexity in the Era of the Data Shake}

Through the analysis of the PoliVisu pilots' direct experience, the article described some particularly relevant challenges related to the increasing complexity of decision making ecosystems and the strategies that public administrations can put in place to take advantage of the data shake. In particular, the causes and effects of the expansion of decision making networks, the involvement of many different actors with specific expertise, and the contextual development of new relationships that bind them were analysed. In this framework, public administrations assume a central role in PoliVisu for the promotion of digital innovation because, thanks to the activities they are involved in, they face new challenges and opportunities providing relevant insights.

On the one hand, it is possible to observe how data can lead to a transformation of decision-makers' activities, effectively supporting every step of the policy cycle, from the design to the evaluation of a policy. Moreover, data allows conceiving the policy making process as an experimental activity in which a greater awareness of current trends described by data can be combined with a renewed ability to manage and retune the implementation of the measures aimed at achieving a specific policy goal. 
On the other hand, this opportunity strongly depends on the ability of public administrations to rethink their role and tasks in the age of digital innovation, following a datacy oriented approach. This process starts with the awareness that the exploitation of data requires an effort to involve multiple actors, both internal and external to the public sector, the structuring of new relationships, and the need to make the internal functioning of the administrative and decision making machine more organic and efficient.

PoliVisu has made it possible to identify these novelties and understand which psychological and structural barriers have been faced by pilots in their path which can keep playing a role in the limited use of data in decision making processes. The analysis of the cases has allowed learning valuable lessons that can help to support the innovative approaches of other administrations outside the project and contribute to the research on digital innovation. For this article's focus, the biggest challenge is the need to build partnerships and relationships both inside and outside the organization and to create the organizational and bureaucratic conditions allowing them to run efficiently.

Considering the internal relations, the pilot cases show that to have an efficient data-supported policy making process, the pilot coordinator (the smart city office) would have benefited from efficient and productive connections with internal actors from all levels (policy makers, operative members) in a regulated framework for the efficient management, sharing and exploitation of available data. Therefore, it is a matter of facilitating all those relations indicated as data and knowledge exchange, internal cooperation for policy making and, if the actors with technical expertise are within the organization, the relations related to data management, analysis, and visualisation. To make these interactions more profitable it is advisable to think in an un-siloed perspective, i.e., breaking the traditional barriers that delimit the different sectors creating cross-cutting collaborative tables starting from the ones that are considered more adapted and readier to a full digital transformation.

At the same time, it is crucial to be aware of the value and usefulness of data in decision making processes, considering them as a precious resource to be wisely collected, managed, and exploited for multiple activities and operations. Both the conditions above must be accompanied by strong political support. An evolution of the governance structure can only be promoted by expressing a strong political will by those who have the power to encourage innovation.

In contrast, external relations introduce a further level of complexity. While internal relations pose an advantage because involved actors know how the organization functions, external relations are based on the participation of new actors who think differently from the public sector. However, PoliVisu has noticed how the construction of private-public partnerships can drive the establishment of efficient collaborations with good advantages for all parties. This opportunity can be seized by overcoming some barriers related to negotiating and defining clear agreements for the collection, use, and management of data. To achieve this outcome, it is paramount to specify which relation to establish with a partner and what to give in return. In line with the evolution of the legal frameworks governing these interactions, the need to build ad hoc procurements to regulate data exchange, access, and ownership, and 
the extent to which external partners can be involved to offer data-based services to public administrations, will be increasingly pressing. In this way, it will be possible to ensure the contractual balance between the different actors involved in data exchange processes and make interactions between all the actors of the ecosystem increasingly efficient and profitable.

Acknowledgements The author thanks Freya Acar, Joran Van Daele and Jonas Verstraete for their valuable contribution and thorough revision of this chapter, and for their support and availability during the stay in Ghent, and Matteo Satta for the hospitality and the precious inputs during the stay in Issy-les-Moulineaux.

\section{References}

Batini C (2018) Datacy, perché una scienza per studiare l'impatto dei dati sulla società in "Agenda Digitale". Available at https://agendadigitale.eu/cittadinanza-digitale/datacy-percheuna-scienza-per-studiare-limpatto-dei-dati-sulla-societa/a. Accessed 5 December 2019

Calzada I (2018) (smart) Citizens from data providers to decision makers? The case study of Barcelona. Sustain 10(9):3252. https://doi.org/10.3390/su10093252

Dente B (2011) Le decisioni di policy. Come si prendono, come si studiano. Il Mulino, Bologna

Kitchin R (2014a) The real-time city? cig data and smart urbanism. GeoJournal 79:1-14

Kitchin R (2014b) The data revolution. big data, open data, data infrastructure \& their consequences. Sage, London

Rabari C, Storper M (2015) The digital skin of cities: urban theory and re-search in the age of the sensored and metered city, ubiquitous computing and big data. Camb J RegNs, Econ Soc 8(1):27-42. https://doi.org/10.1093/cjres/rsu02

Thakuriah P, Tilahun NY, Zellner M (2017) Big data and urban informatics: innovations and challenges to urban planning and knowledge discovery. In: P Thakuriah et al. (eds) Seeing cities through big data. Springer Geography

Wiliford C, Henry C (2012) One culture: computationally intensive research in the humanities and social sciences. Council on Library and Information Resources, Washington DC

Giovanni Lanza PhD student in Urban Planning Design and Policy (UPDP) at the Politecnico di Milano where, in 2017, he obtained his MSc in Urban Planning and Policy Design. Besides his doctoral activities, he has been teaching assistant in Master level courses at Politecnico di Milano since 2017. Through his doctoral research, financed by a thematic scholarship (2019-2022) and developed in close cooperation with the Polivisu project partners, he intends to deepen the opportunities related to the use of big data in the evolving frame of mobility studies and transport planning practice. 
Open Access This chapter is licensed under the terms of the Creative Commons Attribution 4.0 International License (http://creativecommons.org/licenses/by/4.0/), which permits use, sharing, adaptation, distribution and reproduction in any medium or format, as long as you give appropriate credit to the original author(s) and the source, provide a link to the Creative Commons license and indicate if changes were made.

The images or other third party material in this chapter are included in the chapter's Creative Commons license, unless indicated otherwise in a credit line to the material. If material is not included in the chapter's Creative Commons license and your intended use is not permitted by statutory regulation or exceeds the permitted use, you will need to obtain permission directly from the copyright holder.

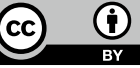

